\title{
High efficiency CCD detectors at UV wavelengths
}

\author{
Erika T. Hamden, ${ }^{a}$ April D. Jewell, ${ }^{b}$ Samuel Gordon, ${ }^{a}$ John Hennessy, ${ }^{b}$ Michael E. Hoenk,${ }^{b}$ \\ Shouleh Nikzad, ${ }^{b}$ David Schiminovich,${ }^{a}$ and D. Christopher Martin ${ }^{c}$ \\ ${ }^{a}$ Columbia University, 550 W 120th St, Pupin Hall, New York, NY 10027, USA; \\ ${ }^{b}$ Jet Propulsion Laboratory, California Institute of Technology, Pasadena, CA 91109, USA; \\ ${ }^{c}$ Department of Astronomy, California Institute of Technology, 1200 East California Blvd, \\ Pasadena, CA 91125, USA;
}

\begin{abstract}
The Faint Intergalactic Redshifted Emission Balloon (FIREBall) is a NASA/CNES balloon-borne ultraviolet multi-object spectrograph designed to observe the diffuse gas around galaxies (the circumgalactic medium) via line emission redshifted to $\sim 205 \mathrm{~nm}$. FIREBall uses a ultraviolet-optimized delta doped e2v CCD201 with a custom designed high efficiency five layer anti-reflection coating. This combination achieves very high quantum efficiency (QE) and photon-counting capability, a first for a CCD detector in this wavelength range. We also present new work on red blocking mirror coatings to reduce red leak.
\end{abstract}

Keywords: Thin Films, Red Blocking, Anti-Reflection Coatings, Ultraviolet, delta doping

\section{INTRODUCTION}

The Faint Intergalactic Red Shifted Emission Balloon (FIREBall-2) is a balloon born UV spectrograph funded jointly by NASA and CNES. FIREBall-2 uses a one meter primary mirror to feed a multi-object spectrograph optimized for narrow-band observations centered around $205 \mathrm{~nm}$. The primary science goal of FIREBall-2 is to observe the diffuse gas around galaxies, known as the circumgalactic medium (CGM), via redshifted line emission. The main targets are emission from Lyman- $\alpha(121.56 \mathrm{~nm})$ at a redshift of $\mathrm{z}=0.7, \mathrm{CIV}$ at $\mathrm{z}=0.3$, and OVI at $\mathrm{z}=1.0$. This emission comes from the cooling of diffuse gas that is gravitationally bound to a galaxy, yet is far enough away from the galaxy to interact with the large scale structure of the universe.

Our current picture of the CGM comes from absorption line studies, using the spectra of background objects, such as quasars, to detect diffuse material around a foreground galaxy. These studies have successfully detected gas and metals around galaxies at a range of redshifts and impact parameters, ${ }^{1-5}$ yet are limited by the small number of background objects per foreground galaxy. With only one or two sightlines per galaxy there is no straightforward way to distinguish between the presence of gas in filaments, a random collection of unconnected gas clumps, or a smooth distribution of gas filling the galactic halo.

FIREBall-2 is designed to overcome these limitations by observing emission directly from the CGM, instead of observing its effect on the spectra of background objects. With a multi-object spectrograph, we can target multiple regions of the CGM around a galaxy or cluster. This approach will provide both spatial and wavelength information about the CGM of a galaxy and will be the start of building a 3D picture of the dynamic CGM. The UV is targeted here for a variety of reasons. A large fraction of expected emission from the CGM (both continuum and line) is emitted at UV wavelengths for both low and high z galaxies. Additionally, almost all emission lines of note for the CGM are emitted at FUV wavelengths. ${ }^{6}$ To observe these lines around a low redshift galaxy requires a space telescope, but a sub-orbital UV telescope can exploit certain wavelengths at a lower cost. There is an atmospheric window of high transmission $(\sim 80 \%)$ between $\mathrm{O}_{2}$ and $\mathrm{O}_{3}$ absorption bands ${ }^{7}$ shown in Figure 1, which allows for observation in the UV at balloon altitudes above ozone and oxygen. FIREBall-2 seeks to exploit this window to observe the CGM in emission at low redshift for the first time.

Further author information: (Send correspondence to Erika T. Hamden)

E-mail: hamden@astro.columbia.edu

Space Telescopes and Instrumentation 2014: Ultraviolet to Gamma Ray, edited by Tadayuki Takahashi,

Jan-Willem A. den Herder, Mark Bautz, Proc. of SPIE Vol. 9144, 91442X · @ 2014 SPIE

CCC code: $0277-786 X / 14 / \$ 18 \cdot$ doi: $10.1117 / 12.2056204$

Proc. of SPIE Vol. $914491442 X-1$ 


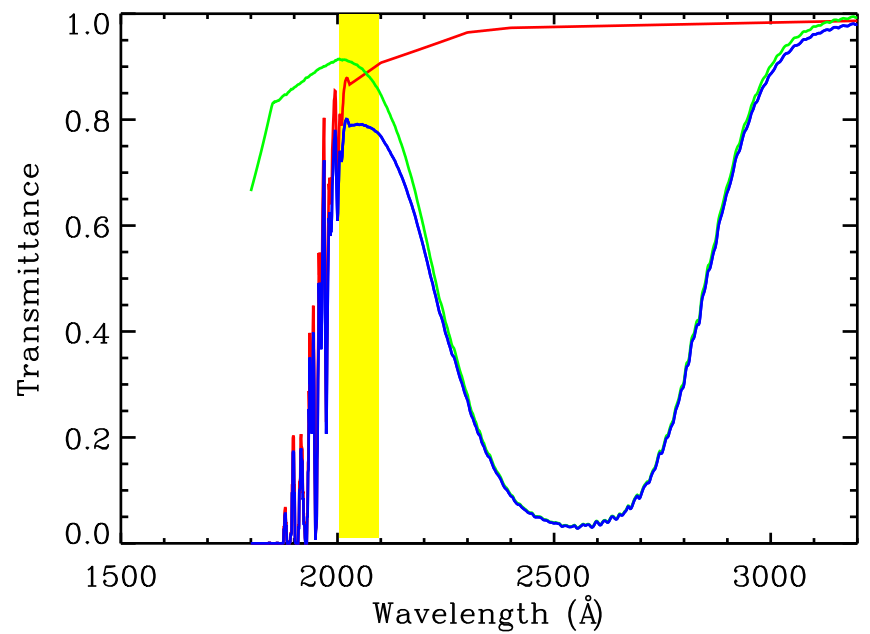

Figure 1. Atmospheric transmission between 100-300 $\mathrm{nm}$ at balloon altitudes. Yellow band shows FIREBall bandpass. The red line shows atmospheric transmission for $\mathrm{O}_{2}$. The green line shows atmospheric transmission for $\mathrm{O}_{3}$. The blue line shows the combined transmission of both $\mathrm{O}_{2}$ and $\mathrm{O}_{3}$, with an $80 \%$ transmission window at around $205 \mathrm{~nm}$. The FIREBall bandpass is designed to fit in this narrow window of high transmission.

\subsection{Changes from FIREBall-1}

A previous version of the mission, FIREBall-1 was launched on two occasions, in 2007 and 2009, from Fort Sumner, New Mexico. ${ }^{8,9}$ FIREBall-1 was a fiber-fed spectrograph which used an Offner design and a GALEXspare near-UV microchannel plate (MCP). ${ }^{10,11}$ FIREBall-1, while a technical and engineering success, recorded data consistent with no detected signal at the 20,000 LU level. This result indicated a need to lower the detection limits for FIREBall-2 and drove the changes made to the current spectrograph and detector. The spectrograph of FIREBall-2 has since been redesigned extensively.

A field corrector expands the field of view from $4 \mathrm{x} 4$ arcmin to $35 \mathrm{x} 11$ arcmin. This increases the number of potential targets per field, up to 50 at a time. The spectrograph itself will use a slit mask instead of fiber bundle. The slit mask, made by NiCoForm of a proprietary nickle cobalt alloy, has a radius of curvature of $125 \mathrm{~mm}$, to perfectly match the curved focal plane from the telescope and field corrector. Laser cutting of the slit mask will be performed at the University of Arizona Instrumentation Center to create precise masks targeting several fields where bright circumgalactic medium emission may be found. The choice to use a slit mask does require the ability to select different masks for different fields of view. This increases the complexity of the system by requiring a rotating stage to select masks. More importantly, however, it increases the overall throughput of the instrument by removing the fiber bundle IFU. The official throughput of the fibers used on FIREBall-1 (Polymicro FVP100110125) was $70 \%{ }^{8}$ While a slit mask does force the selection of only certain targets for observation, and thus has a lower covering fraction, the regions which are observed suffer no loss in throughput. FIREBall-1 conduced a blind survey with a fiber bundle covering the whole field of view. FIREBall-2 more efficiently uses detector area by conducting a targeted survey, where regions with expected CGM emission will be selected in advance. This multiplexing will provide large gains in overall sensitivity.

The detector change from a MCP to a CCD is likewise motivated by a desire to lower the detection limits to below the expected emission levels of the bright CGM. ${ }^{6}$ The MCP used on FIREBall-1 was a GALEX NUV flight spare. This MCP had QE of $6 \%$ at the FIREBall bandpass, operating at high voltage and in a sealed tube. MCPs are known for having very low noise characteristics, ${ }^{12}$ which is vital for observations in this photon-limited regime. Recent improvements to CCD technology have made CCDs a viable alternative to MCPs at some UV wavelengths. These innovations are two-fold, incorporating very high quantum efficiencies at UV wavelengths with the ability to operate in a photon-counting mode with significantly reduced detector noise. These electron multiplying CCDS (EMCCDs) are described in more detail in other works. ${ }^{13-15}$ FIREBall-2 is pioneering the use of EMCCDs in sub-orbital missions, and will use the on-chip gain to minimize noise. We leave a more detailed discussion of this aspect of the detectors to other work. 
We explore in particular the improvements in quantum efficiency beyond that in our recent work ${ }^{16-18}$ in Section 2 and describe additional work for towards increasing throughput via other optical surfaces in Section 3. Finally, we discuss the theoretical overall throughput of FIREBall-2 as compared to FIREBall-1 in Section 4.

\section{ADVANCED ULTRAVIOLET ANTI-REFLECTION COATINGS}

NASA's Jet Propulsion Laboratory (JPL), California Institute of Technology, has had great success in their development of delta doping technology. In standard CCDs, the shallow absorption depth of UV photons in silicon $(<10 \mathrm{~nm})$ requires the use of both back-illuminated and thinned CCDs, to avoid both absorption in the front-side circuitry and recombination losses in the backside surface layer. ${ }^{19}$ Despite these precautions, traps will form in the surface layer between $\mathrm{Si}_{-} \mathrm{SiO}_{2}$, interacting with the shallowly generated charges, causing poor QE, hysteresis, and high dark current. ${ }^{20}$ The insertion of a single atomic layer of heavily doped silicon, deposited using molecular beam epitaxy and capped with pure silicon, passivates the surface layer. This delta doped layer eliminates traps and allows for stable and efficient charge collection within the CCD. ${ }^{21}$ This technique passivates the back surface of a thinned silicon CCD, CMOS, or PIN array and results in nearly $100 \%$ internal QE from the extreme UV to the IR, ${ }^{22}$ yielding reflectance limited QE. While this procedure modifies the internal electric field of the device, the surface itself remains as silicon and its native oxide. Thus, we can create anti-reflection (AR) coatings optimized for this well-defined starting surface.

Our initial work on this subject described the use of single layer AR coatings to improve transmission in delta doped devices. ${ }^{16,17}$ Silicon is highly reflective in the UV $(>60 \%)$ so reducing reflection is a simple way to increase the $\mathrm{QE}$ without modifying the $\mathrm{CCD}$ itself. At visible wavelengths, anti-reflection coatings have been highly refined to produce near $100 \%$ transmission at a selected wavelength. This is achieved through quarterwave stacks and other configurations. ${ }^{23}$ In applying these techniques to UV wavelengths, we began with simple single layer coatings which improved QE to greater than $50 \%$ over a $50 \mathrm{~nm}$ wide bandpass. The materials used were selected in part because of their optical properties (UV transmissive) and easy growth with atomic layer deposition (ALD).

$\mathrm{HfO}_{2}, \mathrm{Al}_{2} \mathrm{O}_{3}, \mathrm{SiO}_{2}$, and $\mathrm{MgF}_{2}$ were all used in single layer coatings, achieving greater than $50 \%$ QE between $130 \mathrm{~nm}$ and $350 \mathrm{~nm} .{ }^{17}$ We reported on work with more complex coatings in $2012,{ }^{18}$ including initial modeling of three and five layer films. These models were optimized for use at $155 \mathrm{~nm}, 205 \mathrm{~nm}$, and $255 \mathrm{~nm}$ as an example of the range in peak wavelengths, although in principle they can be created for nearly any wavelength above 130 $\mathrm{nm}$. We also discussed initial work on films specifically optimized for the FIREBall band pass. As discussed briefly in Section 1, the FIREBall bandpass is set by atmospheric transmission between $\mathrm{O}_{2}$ and $\mathrm{O}_{3}$ absorption bands. The transmission peak at $205 \mathrm{~nm}$ and bandwidth of $\sim 10 \mathrm{~nm}$ set the parameters for all optical coatings under consideration.

Since 2012, we have improved both the deposition techniques and the exact model to achieve a high quality, high QE coating optimized for $>80 \% \mathrm{QE}$ at the FIREBall bandpass. This coating contains five alternating layers of $\mathrm{Al}_{2} \mathrm{O}_{3}$ and $\mathrm{SiO}_{2}$, although we are exploring more complex coatings for future uses.

\subsection{Modeling, Deposition, and Testing}

All coatings were modeled using TFcalc (Software Spectra, Portland, OR, USA). Optical constants come from Palik, ${ }^{24,25}$ although in some cases the constants used are from thin films deposited using ALD at JPL and measured by J.A. Woollam using vacuum ellipsometry. These constants, including those for delta doped silicon, will be published in a paper currently in preparation. We choose to focus on models using only $\mathrm{Al}_{2} \mathrm{O}_{3}$ and $\mathrm{SiO}_{2}$ in part because their growth is straightforward, well characterized, and repeatable using $\mathrm{ALD} . \mathrm{Al}_{2} \mathrm{O}_{3}$ has been used as a barrier layer in previous work ${ }^{17}$ and is a reliable first layer for a delta doped device.

All coatings were deposited using ALD, at NASA's Jet Propulsion Laboratory. ALD enables conformal, atomic level control over film growth and allows well controlled, repeatable depositions. ALD recipes for $\mathrm{Al}_{2} \mathrm{O}_{3}$ came from Goldstein $(2008)^{26}$ and $\mathrm{SiO}_{2}$ from Dingemans (2011). ${ }^{27}$ Often growth recipes required modifications to accommodate the equipment used. Modified growth recipes were typically validated using either X-ray photo-electron spectroscopy (XPS), transmission electron microscopy (TEM), or both. Growths were typically conducted at $200{ }^{\circ} \mathrm{C}$. More information on ALD growth techniques can be found in Greer et al., (2013). ${ }^{28}$ 


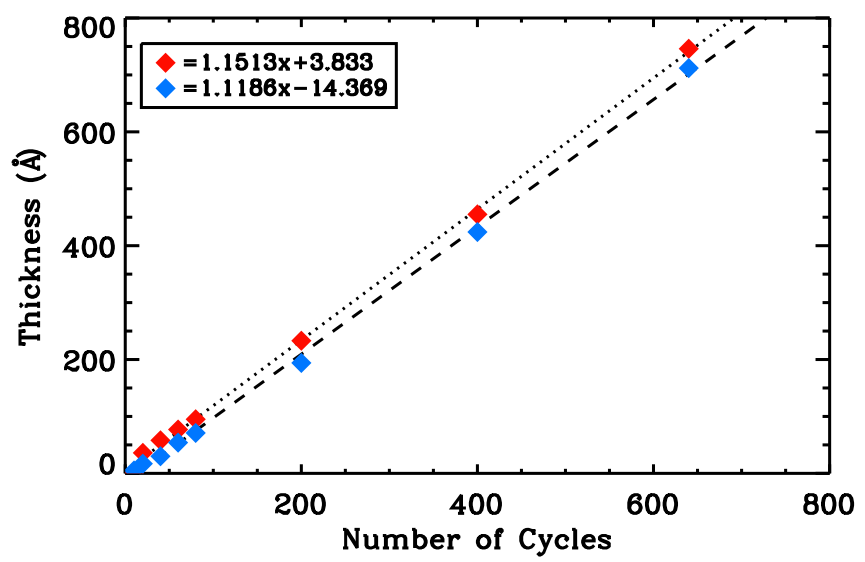

Figure 2. Growth rate of $\mathrm{SiO}_{2}$ on both silicon (in red) and on a $7 \mathrm{~nm}$ thick $\mathrm{Al}_{2} \mathrm{O}_{3}$ base layer (in blue). While the growth per cycle remains constant, the initial growth is much slower on the $\mathrm{AL}_{2} \mathrm{O}_{3}$ base layer, reflecting the difference in the number of cycles required for good surface nucleation.

For initial reflectance tests, films were grown on one inch $<100>1-20 \mathrm{Ohm}$-cm silicon wafers (to test reflectance) and on fused silica windows (to test absorption). Tests were conducted using a reflectance set-up at Columbia University. The samples were placed in a vacuum chamber maintained at less than $1 \times 10^{-4}$ torr for the duration of the measurement. An Acton monochrometer fed by a focused deuterium lamp provides light from $120 \mathrm{~nm}$ to $450 \mathrm{~nm}$. The light is then reflected off of samples at any angle of incidence from nearly normal $\left(5^{\circ}\right)$ to straight through, in the case of transparent substrates. In testing films grown on silicon substrates, reflectance was measured at $5-10^{\circ}$ from normal. For transparent substrates, usually fused silica, the light was able to pass directly through the sample. Current measurements were taken using a PMT (R6095) with a scintillator and light pipe assembly (McPherson Model 658).

\section{$2.2 \mathrm{SiO}_{2}$ growth rates}

In creating these multilayers we had to contend, for the first time, with film deposition on substrates other than silicon. During our initial work, we found that our overall layer thickness was consistently too thin, pushing our peak transmission to a lower wavelength $(\sim 190 \mathrm{~nm})$ instead of the desired $205 \mathrm{~nm}$. Upon further investigation, it was apparent that the layers of $\mathrm{SiO}_{2}$ were generally 3-4 nm thinner than expected. Since $\mathrm{Al}_{2} \mathrm{O}_{3}$ is the first layer to be deposited in our coatings, the second layer $\left(\right.$ of $\left.\mathrm{SiO}_{2}\right)$ must be grown on this $\mathrm{Al}_{2} \mathrm{O}_{3}$ base. The growth rates for $\mathrm{ALD} \mathrm{SiO}_{2}$ were calibrated with $\mathrm{Si}$ substrates, and so needed to be remeasured on $\mathrm{Al}_{2} \mathrm{O}_{3}$ substrates. The actual growth rate ( $\AA$ deposited per number of ALD cycles) is almost exactly the same, regardless of substrate. But the discrepancy in final thickness is due to the number of additional ALD cycles required for nucleation of the film on $\mathrm{Al}_{2} \mathrm{O}_{3}$ vs. a silicon substrate. Figure 2 shows the effect of these two different substrates on the final $\mathrm{SiO}_{2}$ film thickness.

Once this nucleation difference was accounted for, we were able to grow multi-layer films of $\mathrm{Al}_{2} \mathrm{O}_{3}$ and $\mathrm{SiO}_{2}$ with good accuracy. Futher work on calibrating other materials on $\mathrm{Al}_{2} \mathrm{O}_{3}$ or other non-silicon substrates remains to be completed.

\subsection{5 layer coating}

Figure 3 shows the expected transmission for an early version of the five layer film of $\mathrm{SiO}_{2}$ and $\mathrm{Al}_{2} \mathrm{O}_{3}$. Peak transmission is at $190 \mathrm{~nm}$, reflecting the thinner $\mathrm{SiO}_{2}$ layers before the growth rate correction. This transmission is determined by subtracting both the measured reflectance and the calculated absorption from $100 \%$. Reflectance on a silicon substrate, shown in the top right, is measured as described in Section 2.1 and follows the model extremely well. Both reflectance and transmittance are measured for the film on a fused silica window. These measurements, which also follow the model well, confirm that the behavior of the film is as expected on this alternative substrate. To calculate absorption in the layer, we subtract the measured absorption of an uncoated window from the measured absorption of the layer and window. The difference in absorption we ascribe to the 

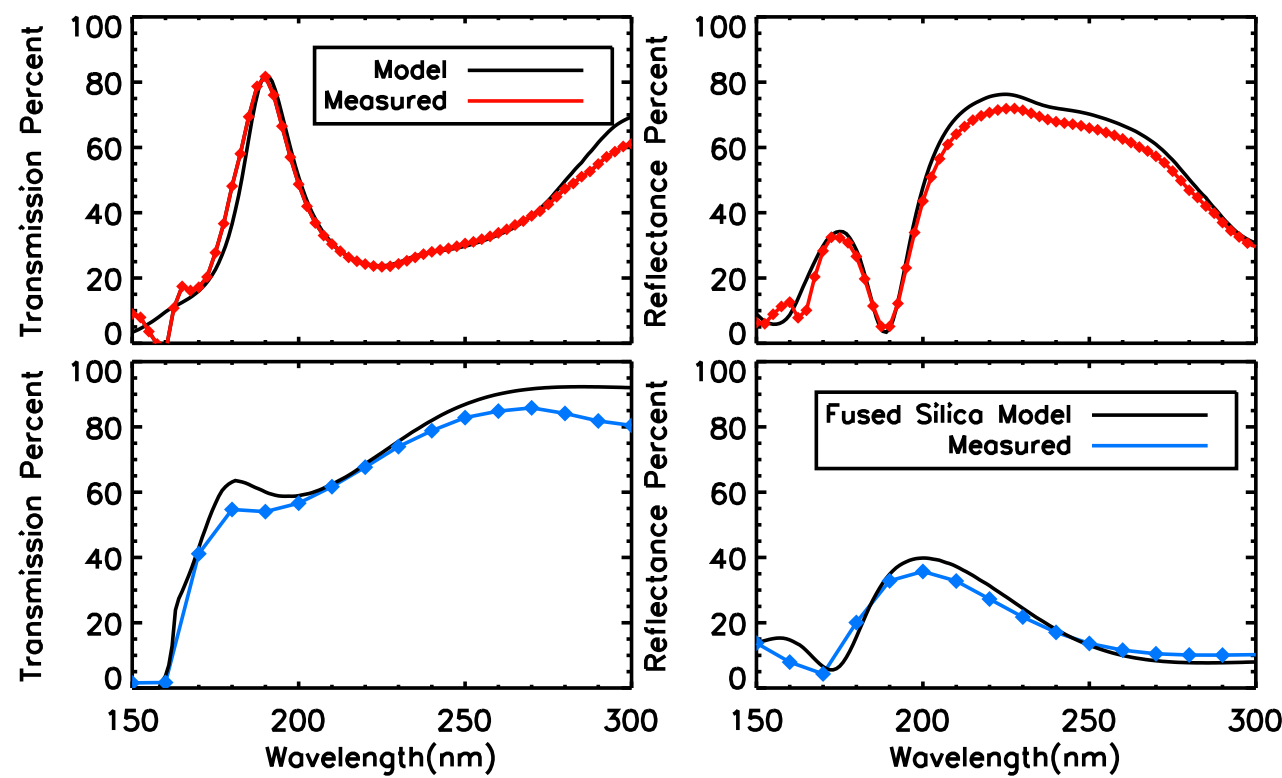

Figure 3. Top Left Estimated transmission for a five layer film of $\mathrm{SiO}_{2}$ and $\mathrm{Al}_{2} \mathrm{O}_{3}$. Peak transmission is at 190 nm, and far exceeds previous work at this wavelength (old record was approximately $60 \%$ transmission). This transmission was determined by subtracting the reflectance and absorption from $100 \%$. Top Right Measured reflectance for a five layer film of $\mathrm{SiO}_{2}$ and $\mathrm{Al}_{2} \mathrm{O}_{3}$. Minimum reflectance is at $190 \mathrm{~nm}$. Bottom Left Measured transmission for five layer film on fused silica window. Bottom Right Measured reflectance for five layer film on fused silica window. Both transmission and reflectance of the 11 layer film on fused silica were used to calculate expected absorption from the film itself, as described in Section 2.3. This absorption measurement is combined with reflectance measurements (Top Right) to calculate expected transmission (Top Left) from the film when deposited on a silicon device.

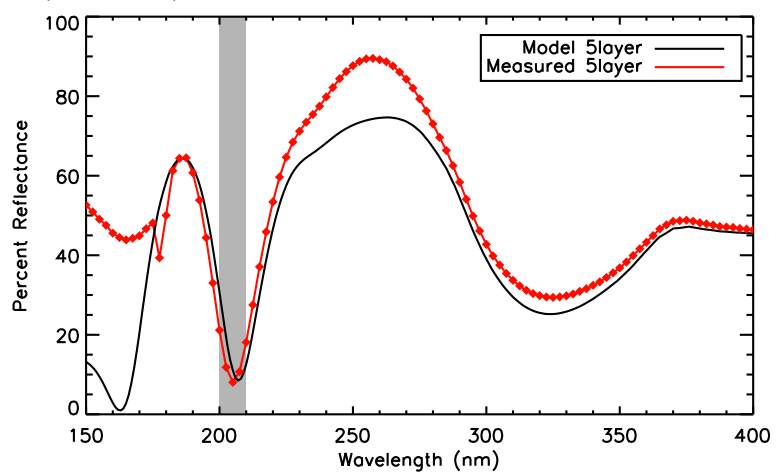

Figure 4. Measured reflectance of improved five layer film design, grown using new $\mathrm{SiO}_{2}$ deposition rates. Minimum reflectance is at $205 \mathrm{~nm}$, with good fidelity to the model.

effect of the layer. This underestimates the absorption at very low wavelengths $(<160 \mathrm{~nm})$, where the fused silica is very absorbing. However, since our region of interest is at $200 \mathrm{~nm}$ and longer, we don't expect this will significantly change our results. For shorter wavelength coatings, a $\mathrm{MgF}_{2}$ window would be advisable. Estimated transmission is calculated by subtracting this calculated absorption from a fused silica substrate and measured reflectance off a silicon substrate from $100 \%$.

Figure 4 shows only the reflectance measurement for a film grown with re-calibrated $\mathrm{SiO}_{2}$ growth rates. This film has been deposited on delta doped devices for QE testing. The testing set-up is described in greater detail in Jacquot et al., $(2011)^{29}$ and was used to measure QE for our previous work on single layer AR coatings. ${ }^{17}$ Testing is ongoing on thinned and delta doped e2v CCD 201s, both with and without the five layer FIREBall optimized coating. 


\begin{tabular}{|c|c|c|c|c|}
\hline Name & No. Layers & $\begin{array}{c}\text { Mean R } \\
\text { In Band }\end{array}$ & $\begin{array}{c}\text { Mean R } \\
\text { Out of Band }\end{array}$ & $\begin{array}{c}\text { Angles } \\
\text { (deg) }\end{array}$ \\
\hline RB A 1 & 18 & 87.7 & 5.3 & $0-7$ \\
RB A 2 & 18 & 87.8 & 5.2 & $0-7$ \\
RB B 1 & 24 & 92.1 & 4.0 & $15-23$ \\
RB B 2 & 22 & 92.1 & 4.0 & $15-23$ \\
RB C 1 & 13 & 83.7 & 6.1 & $0-25$ \\
RB C 2 & 19 & 90.3 & 4.9 & $0-25$ \\
RB C 3 & 28 & 95.1 & 4.8 & $0-25$ \\
\hline
\end{tabular}

Table 1. Characteristics of red blocking filters designed for use on FIREBall spectrograph optics. A films are designed for angles of incidence to Schmidt mirrors in spectrograph, while $\mathbf{B}$ films are designed to be used on the folding flats. $\mathbf{C}$ films can be applied to any spectrograph optic. All films are made of $\mathrm{HfO}_{2}, \mathrm{Al}_{2} \mathrm{O}_{3}$, and $\mathrm{SiO}_{2}$. Mean reflectance in band is calculated from $197-212 \mathrm{~nm}$, while mean reflectance out of band calculated from $280-800 \mathrm{~nm}$.

\section{RED BLOCKING COATINGS}

FIREBall-1 used a dichroic to direct visible light to a guidance system and the UV output to the spectrograph. MCPs have some red sensitivity, so the dichroic also acted as a red blocking filter. FIREBall-2 will instead use a reflective slit-mask to direct light from non-science targets to the guidance system. Instead of a dichroic, out of band light will be rejected via the optical coatings on the surface of the spectrograph mirrors. The main function of the coating is to minimize out of band light and reduce red leak, while not sacrificing in-band reflectance. Here we require $<10 \%$ reflectance out of band and $>85 \%$ reflectance in band. Between 220 and $\sim 280 \mathrm{~nm}$, the atmosphere is very absorbing, and will remove those wavelengths without any assistance from us. Thus, we focus our rejection efforts on wavelengths longer than $280 \mathrm{~nm}$. These coatings are very similar to traditional dichroics, passing long wavelength light and reflecting short wavelength light. Here we apply the coatings directly to a curved optical surface instead of using an additional optical element.

In developing potential red blocking coatings, we have relied on a relatively simple construction. The red blocking coating consists of two distinct sections grown on a fused silica substrate. The section adjacent to the substrate will be as transmissive as possible at all wavelengths, but with emphasis on wavelengths above 250 $\mathrm{nm}$. This coating section serves to reduce out of band reflectance. A second section will then be applied which increases reflectance only in the desired band. This two phase structure serves to create highly reflective films in band without excessive out of band light. It also allows more freedom in our choice of materials. With UV light reflected off of the upper section, we are free to incorporate UV absorbing materials in the lower section. To prevent visible light from reflecting off of the back surface of the optic and re-entering the optical path, we will apply a highly absorbing layer, potentially some optical black, to the backside of the optic. This layer will absorb any transmitted light that passes through the optic.

We have developed a suite of red blocking coatings optimized for the FIREBall bandpass. These coatings were designed to be applied to fused silica surfaces, but could easily be redesigned for almost any type of glass. The upper, reflective section is much less sensitive to the underlying substrate, and the lower section can be redesigned to accommodate the variations in optical properties between different glass types. The coatings we have developed are designed to be highly reflective over a range of angles of incidence (roughly $0-25^{\circ}$ for all four spectrograph optics). In practice only the first two spectrograph optics will be coated. The simplest of these coatings uses $\mathrm{HfO}_{2}, \mathrm{Al}_{2} \mathrm{O}_{3}$, and $\mathrm{SiO}_{2}$ in a 13 layer stack, while the most complex uses 28 layers. These coatings are summarized in Table 1, and an example of reflectance for the most complex coating is shown in Figure 5, while the simplest such coating is shown in Figure 6. Other materials can also be used to create these types of coatings, but we selected these initial materials based on ease of growth using ALD.

We applied the simplest red blocking coating (RB-C-1) to a glass slide for initial tests, using ALD as described above. The reflectance results are shown in Figure 6. Both the model and the reflectance results are plotted. These samples were tested as described in Section 2.1. The results match the model to a good degree, with differences mainly attributable to a glass substrate instead of a fused silica substrate. One other consideration is the growth rate of $\mathrm{HfO}_{2}$ on an $\mathrm{Al}_{2} \mathrm{O}_{3}$ base layer (and vice versa), which has not yet been accounted for in the 

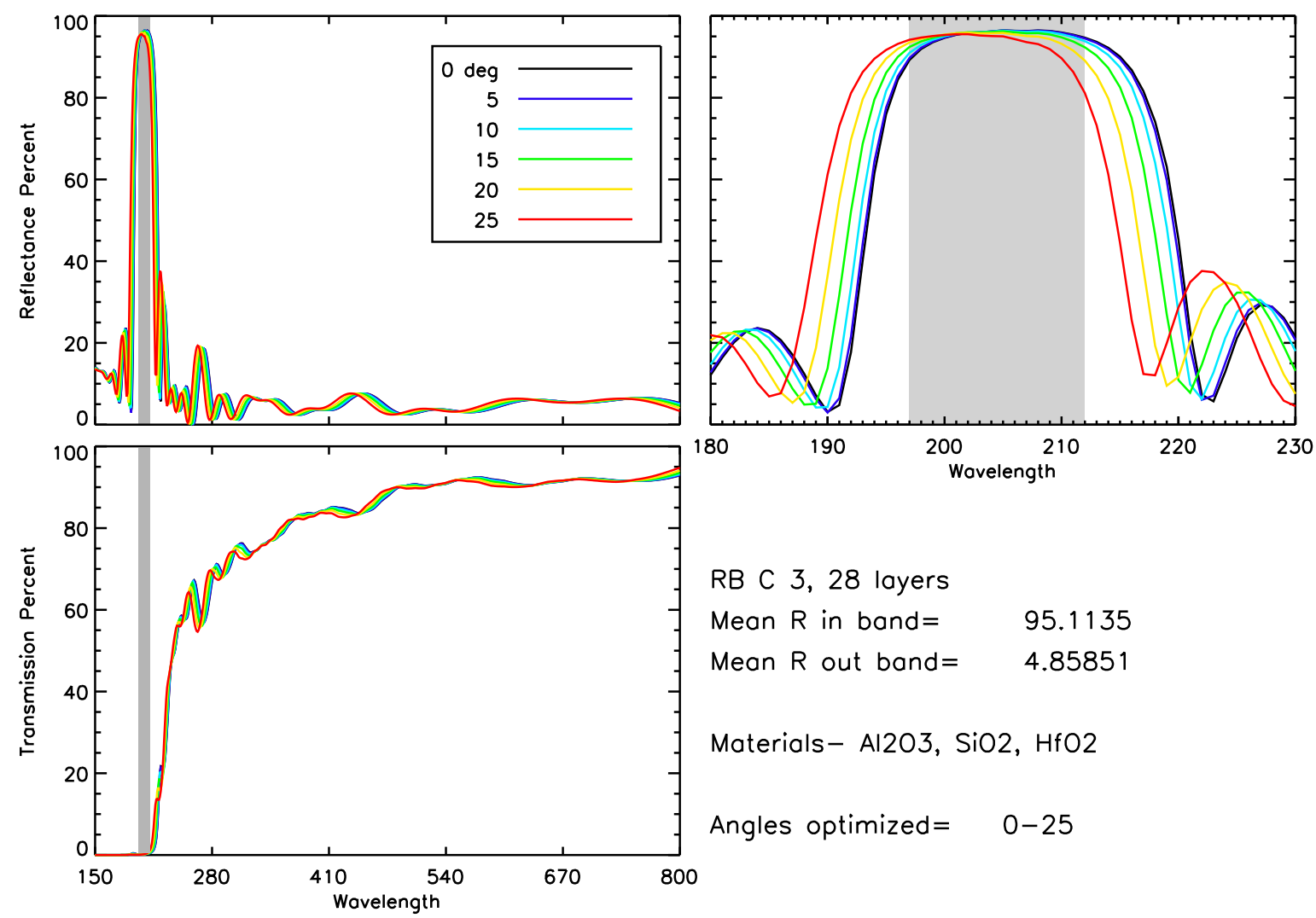

RB C 3, 28 layers

Mean $\mathrm{R}$ in band=

95.1135

Mean $\mathrm{R}$ out band=

4.85851

Materials- $\mathrm{Al} 2 \mathrm{O} 3, \mathrm{SiO} 2, \mathrm{HfO} 2$

Angles optimized $=0-25$

Figure 5. Example of most complex red blocking design for FIREBall-2. This design (RB-C-3) uses 28 layers of $\mathrm{HfO}_{2}$, $\mathrm{Al}_{2} \mathrm{O}_{3}$, and $\mathrm{SiO}_{2}$, and is designed for high reflectivity at all angles of incidence in the spectrograph. Upper Left: Reflectance vs. wavelength for a range of angles of incidence. The FIREBall band is indicated in gray. Upper Right: Close up of reflectance for the FIREBall bandpass. The change with angle of incidence is more clear in this case. Lower Left: Transmission vs. wavelength for a range of angles of incidence. The light transmitted through the optic will be absorbed by an optical black at the back of the optic.

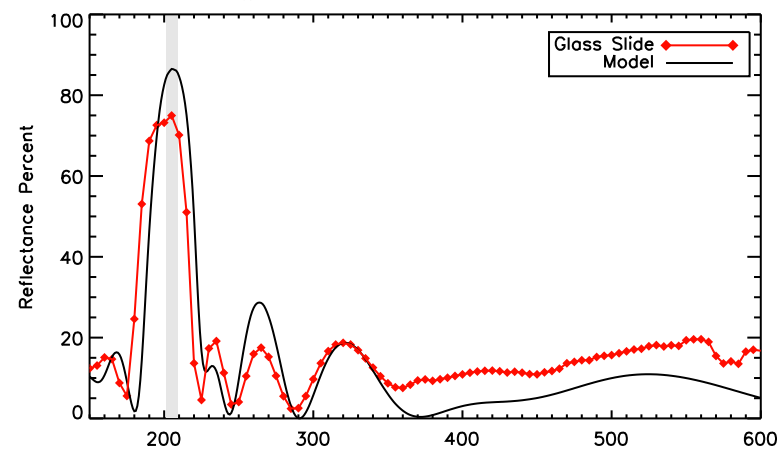

Figure 6. Reflectance measurement of red blocking coating RB-C-1 on a glass slide with a $5^{\circ}$ angle of incidence. The FIREBall band is highlighted in gray. The differences in peak height are possibly due to two things. The first is that the model has a fused silica substrate while the actual coating was grown on a glass slide. The second is there may be growth rate discrepancies for $\mathrm{HfO}_{2}$ on $\mathrm{Al}_{2} \mathrm{O}_{3}$ that we haven't fully explored.

manner describe in Section 2.2 for $\mathrm{SiO}_{2}$. This work shows the promise in such coatings for use in the future. We plan on conducting additional tests with fused silica and other glass substrates. 


\section{FIREBALL SENSITIVITY}

The set of coatings described here includes a coating applied directly to the detector and a set of coatings applied to two optics in the spectrograph. Each coating has been designed to maximize throughput in the FIREBall band while rejecting redder wavelengths. Additional contributors to the overall throughput are the atmospheric transmission (which depends very strongly on altitude), other optics (telescope and field corrector), and finally the grating. With these factors in mind, we estimate overall throughput of FIREBall, shown in Figure 7.

We assume $90 \%$ reflectance at all wavelengths on optics without an optimized coating, and $50 \%$ efficiency in the grating. The average throughput is $13 \%$ from 200 to $210 \mathrm{~nm}$, a large improvement over the $0.5 \%$ system efficiency from FIREBall-1. ${ }^{8}$ The main contributors to the higher throughput of FIREBall-2 is the detector, improved grating, and replacement of the fiber bundle with a slit mask. This calculation does not take into account the overall fill factor, given that the slit mask will block a fraction of the field of view. The losses due to the slit mask blocking are offset by the targeted nature of the CGM survey, yielding more useful targets per field of view.

\subsection{Redleak}

One benefit of using a microchannel plate as a detector is the very strong efficiency cutoff at longer wavelengths. MCPs have been described as "solar-blind" because of their comparatively low sensitivity at visible wavelengths. One concern in using CCD detectors is that they are quite good at detecting visible light, usually with higher QE in the visible than the UV. Even with our high efficiency AR coating, visible QE is greater than $50 \%$ at wavelengths longer than $300 \mathrm{~nm}$. The design of our red blocking films, described in Section 3 is a first step in mitigating this issue. Two bounces off of the red blocking film suppresses the visible light to $0.3 \%$ of the initial value, averaged from 280 to $800 \mathrm{~nm}$. Furthermore, since FIREBall is a spectrograph the wavelength dispersion of the grating also minimizes out of band light reaching the detector. With both of these red leak suppressing factors, out of band light should be reduced significantly.

The conventional wisdom regarding just how important red leak is for a UV telescope may be overstated. Commonly quoted calculations regarding the number of visible photons that must be rejected for every UV photon detected have not taken into account the type of objects commonly observed with a UV telescope. Observing a very red, cool object, such as in searching for planets around dwarf stars or observing elliptical galaxies, will naturally have an abundance of visible photons and a paucity of UV photons. ${ }^{30,31}$ But many UV observations will not target these objects, instead focusing on things which are in fact bright in the UV. Such objects typically have a range in FUV/Vis ratio between 1 and .001 (1 to 1000 visible photons per FUV photons). ${ }^{32}$ Furthermore, these calculations fail to take into account differences in sky background between the FUV and visible. The FUV has significantly lower zodiacal contamination than the visible and lower sky

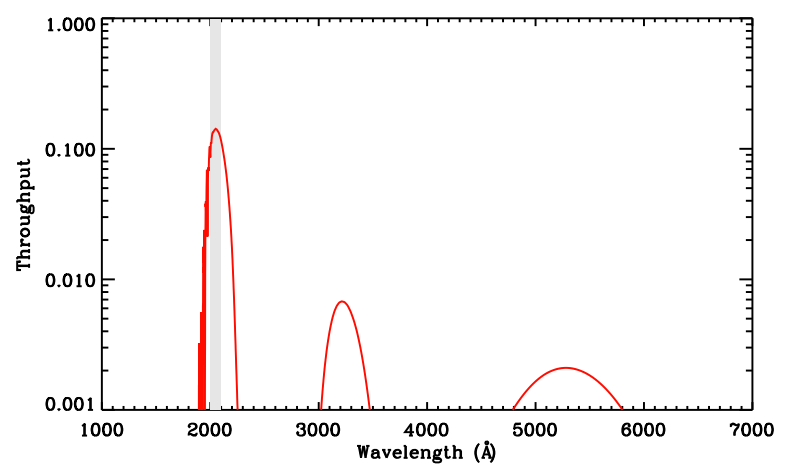

Figure 7. Theoretical estimate of overall FIREBall throughput, with a log scale. Includes atmospheric transmission, siderostat, primary mirror, two field corrector mirrors, two spectrograph optics with red blocking coating (RB C 1), grating with $50 \%$ efficiency, two spectrograph optics with aluminum plus $\mathrm{MgF}_{2}$, detector with high efficiency AR coating. Throughput is above $10 \%$ between 200 and $210 \mathrm{~nm}$, and nearly $14 \%$ at $205 \mathrm{~nm}$. Out of band rejection is better than 1 in 20 between 300 and $350 \mathrm{~nm}$, and better than 1 in 50 elsewhere. These ratios are low enough to ensure minimal contamination from redder wavelengths provided our grating meets the scattering requirement. 
backgrounds overall than the visible. The observations we are conducting will be background limited, and the $10^{6}-10^{8}$ numbers don't take that difference into account.

Finally, the constraints on red leak for a spectrograph are quite different than for an imager. A spectrograph with a single grating will reject out of band light by $10^{-3}$, with the main concern being scattering off of the grating. With the addition of red blocking filters, like those described above, any contribution from out of band light is well below the expected sky background level.

FIREBall-2 will launch in the fall of 2015 from Fort Sumner, New Mexico.

\section{ACKNOWLEDGMENTS}

The research was carried out in part at the Jet Propulsion Laboratory, California Institute of Technology, under a contract with NASA. This work was partially supported by KISS, the W. M. Keck Institute for Space Studies, and by NASA Headquarters under the NASA Earth and Space Science Fellowship Program, NASA Grant NNX11AO07H, and NASA Grant NNX12AF29G. This research was supported in part through an appointment to the NASA Postdoctoral Program at the Jet Propulsion Lab, administered by Oak Ridge Associated Universities through a contract with NASA.

\section{REFERENCES}

[1] Hennawi, J. F., Prochaska, J. X., Burles, S., Strauss, M. A., Richards, G. T., Schlegel, D. J., Fan, X., Schneider, D. P., Zakamska, N. L., Oguri, M., Gunn, J. E., Lupton, R. H., and Brinkmann, J., "Quasars Probing Quasars. I. Optically Thick Absorbers near Luminous Quasars," Astrophysical Journal 651, 61-83 (Nov. 2006).

[2] Ribaudo, J., Lehner, N., Howk, J. C., Werk, J. K., Tripp, T. M., Prochaska, J. X., Meiring, J. D., and Tumlinson, J., "Evidence for Cold Accretion: Primitive Gas Flowing onto a Galaxy at z 0.274," Astrophysical Journal 743, 207 (Dec. 2011).

[3] Steidel, C. C., Bogosavljević, M., Shapley, A. E., Kollmeier, J. A., Reddy, N. A., Erb, D. K., and Pettini, M., "Diffuse Ly $\alpha$ Emitting Halos: A Generic Property of High-redshift Star-forming Galaxies," Astrophysical Journal 736, 160 (Aug. 2011).

[4] Thom, C., Tumlinson, J., Werk, J. K., Prochaska, J. X., Oppenheimer, B. D., Peeples, M. S., Tripp, T. M., Katz, N. S., O'Meara, J. M., Ford, A. B., Davé, R., Sembach, K. R., and Weinberg, D. H., "Not Dead Yet: Cool Circumgalactic Gas in the Halos of Early-type Galaxies," Astrophysical Journal, Letters 758, L41 (Oct. 2012).

[5] Tumlinson, J., Thom, C., Werk, J., Prochaska, J. X., Tripp, T., Katz, N., Dave, R., Oppenheimer, B. D., Meiring, J., Brady Ford, A., O'Meara, J., Peeples, M., Sembach, K., and Weinberg, D., "The COS-Halos Survey: Rationale, Design, and A Census of Circumgalactic Neutral Hydrogen," ArXiv e-prints (Sept. 2013).

[6] Bertone, S., Aguirre, A., and Schaye, J., "How the diffuse Universe cools," Monthly Notices of the Royal Astronomical Society 430, 3292-3313 (Apr. 2013).

[7] Brewer, A. W. and Wilson, A. W., "Measurements of solar ultraviolet radiation in the stratosphere," Quarterly Journal of the Royal Meteorological Society 91, 452-461 (Oct. 1965).

[8] Tuttle, S. E., Schiminovich, D., Milliard, B., Grange, R., Martin, D. C., Rahman, S., Deharveng, J., McLean, R., Tajiri, G., and Matuszewski, M., "The FIREBall fiber-fed UV spectrograph," in [Society of Photo-Optical Instrumentation Engineers (SPIE) Conference Series], Presented at the Society of PhotoOptical Instrumentation Engineers (SPIE) Conference 7014 (Aug. 2008).

[9] Milliard, B., Martin, D. C., Schiminovich, D., Evrard, J., Matuszewski, M., Rahman, S., Tuttle, S., McLean, R., Deharveng, J.-M., Mirc, F., Grange, R., and Chave, R., "FIREBALL: the Faint Intergalactic medium Redshifted Emission Balloon: overview and first science flight results," in [Society of Photo-Optical Instrumentation Engineers (SPIE) Conference Series], Society of Photo-Optical Instrumentation Engineers (SPIE) Conference Series 7732 (July 2010). 
[10] Doliber, D. L., Stock, J. M., Jelinsky, S. R., Malloy, J., Jelinsky, P. N., Siegmund, O. H., and Hull, J. S., "Development challenges for the GALEX UV sealed tube detectors," in [UV, Optical, and IR Space Telescopes and Instruments], Breckinridge, J. B. and Jakobsen, P., eds., Society of Photo-Optical Instrumentation Engineers (SPIE) Conference Series 4013, 402-410 (July 2000).

[11] Tuttle, S. E., Schiminovich, D., Matuszewski, M., Rahman, S., McLean, R., Martin, C., Frank, S., Milliard, B., and Deharveng, J., "FIREBall: Initial Science Results from the First UV Fiber-fed Integral Field Spectrograph," in [American Astronomical Society Meeting Abstracts No. 215], Bulletin of the American Astronomical Society 42, 315.06 (Jan. 2010).

[12] Morrissey, P., Schiminovich, D., Barlow, T. A., Martin, D. C., Blakkolb, B., Conrow, T., Cooke, B., Erickson, K., Fanson, J., Friedman, P. G., Grange, R., Jelinsky, P. N., Lee, S.-C., Liu, D., Mazer, A., McLean, R., Milliard, B., Randall, D., Schmitigal, W., Sen, A., Siegmund, O. H. W., Surber, F., Vaughan, A., Viton, M., Welsh, B. Y., Bianchi, L., Byun, Y.-I., Donas, J., Forster, K., Heckman, T. M., Lee, Y.-W., Madore, B. F., Malina, R. F., Neff, S. G., Rich, R. M., Small, T., Szalay, A. S., and Wyder, T. K., "The On-Orbit Performance of the Galaxy Evolution Explorer," The Astrophysical Journal Letters 619, L7-L10 (Jan. 2005).

[13] Mackay, C. D., Tubbs, R. N., Bell, R., Burt, D. J., Jerram, P., and Moody, I., "Subelectron read noise at MHz pixel rates," in [Society of Photo-Optical Instrumentation Engineers (SPIE) Conference Series], M. M. Blouke, J. Canosa, \& N. Sampat, ed., Presented at the Society of Photo-Optical Instrumentation Engineers (SPIE) Conference 4306, 289-298 (May 2001).

[14] Jerram, P., Pool, P. J., Bell, R., Burt, D. J., Bowring, S., Spencer, S., Hazelwood, M., Moody, I., Catlett, N., and Heyes, P. S., "The LLCCD: low-light imaging without the need for an intensifier," in [Sensors and Camera Systems for Scientific, Industrial, and Digital Photography Applications II], Blouke, M. M., Canosa, J., and Sampat, N., eds., Society of Photo-Optical Instrumentation Engineers (SPIE) Conference Series 4306, 178-186 (May 2001).

[15] Gach, J., Guillaume, C., Boissin, O., and Cavadore, C., "First Results of aN L3CCD in Photon Counting Mode," in [Scientific Detectors for Astronomy, The Beginning of a New Era], P. Amico, J. W. Beletic, \& J. E. Belectic, ed., Astrophysics and Space Science Library 300, 611-614 (2004).

[16] Hamden, E. T., Greer, F., Hoenk, M. E., Blacksberg, J., Dickie, M. R., Nikzad, S., Martin, D. C., and Schiminovich, D., "Ultraviolet antireflection coatings for use in silicon detector design," Applied Optics 50, 4180-4188 (July 2011).

[17] Nikzad, S., Hoenk, M. E., Greer, F., Jacquot, B., Monacos, S., Jones, T. J., Blacksberg, J., Hamden, E., Schiminovich, D., Martin, C., and Morrissey, P., "Delta-doped electron-multiplied ccd with absolute quantum efficiency over $50 \%$ in the near to far ultraviolet range for single photon counting applications," Appl. Opt. 51, 365-369 (Jan 2012).

[18] Hamden, E. T., Greer, F., Schiminovich, D., Nikzad, S., and Martin, D. C., "UV photon-counting CCD detectors that enable the next generation of UV spectroscopy missions: AR coatings that can achieve 8090\% QE," in [Society of Photo-Optical Instrumentation Engineers (SPIE) Conference Series], Society of Photo-Optical Instrumentation Engineers (SPIE) Conference Series 8453 (July 2012).

[19] Crowell, M. H., Buck, T. M., Labuda, E. F., Dalton, J. V., and Walsh, E. J., "A camera tube with a silicon diode array target," Bell System Technical Journal 46(2), 491-495 (1967).

[20] Janesick, J., Elliott, T., Frasehetti, G., Collins, S., Blouke, M., and Corrie, B., "Charge-coupled device pinning technologies," (1989).

[21] Nikzad, S., Hoenk, M. E., Grunthaner, P. J., Terhune, R. W., Grunthaner, F. J., Winzenread, R., Fattahi, M. M., Tseng, H.-F., and Lesser, M. P., "Delta-doped CCDs: high QE with long-term stability at UV and visible wavelengths," in [Society of Photo-Optical Instrumentation Engineers (SPIE) Conference Series], Crawford, D. L. and Craine, E. R., eds., Society of Photo-Optical Instrumentation Engineers (SPIE) Conference Series 2198, 907-915 (June 1994).

[22] Hoenk, M. E., Grunthaner, P. J., Grunthaner, F. J., Terhune, R. W., Fattahi, M., and Tseng, H.-F., "Growth of a delta-doped silicon layer by molecular beam epitaxy on a charge-coupled device for reflection-limited ultraviolet quantum efficiency," Applied Physics Letters 61, 1084-1086 (Aug. 1992).

[23] MacLeod, H. A., [Thin-Film Optical Filters], London, GB: Institute of Physics Publishing (1986). 
[24] Palik, E. D., [Handbook of optical constants of solids] (1985).

[25] Palik, E. D., [Handbook of optical constants of solids II] (1991).

[26] Goldstein, D. N., McCormick, J. A., and George, S. M., "Al2O3 Atomic Layer Deposition with Trimethylaluminum and Ozone Studied by in Situ Transmission FTIR Spectroscopy and Quadrupole Mass Spectrometry," Journal of Physical Chemistry 112, 19530-19539 (Nov. 2008).

[27] Dingemans, G., Helvoirt, C. V., de Sanden, M. V., and Kessels, W. M., "Plasma-assisted atomic layer deposition of low temperature sio[sub 2]," ECS Transactions 35(4), 191-204 (2011).

[28] Greer, F., Hamden, E., Jacquot, B. C., Hoenk, M. E., Jones, T. J., Dickie, M. R., Monacos, S. P., and Nikzad, S., "Atomically precise surface engineering of silicon ccds for enhanced uv quantum efficiency," Journal of Vacuum Science Technology A: Vacuum, Surfaces, and Films 31, 01A103-01A103-9 (jan 2013).

[29] Jacquot, B. C., Monacos, S. P., Hoenk, M. E., Greer, F., Jones, T. J., and Nikzad, S., "A system and methodologies for absolute quantum efficiency measurements from the vacuum ultraviolet through the near infrared," Review of Scientific Instruments 82, 043102 (Apr. 2011).

[30] O'Connell, R. W., "Far-Ultraviolet Radiation from Elliptical Galaxies," Annual Review of Astron and Astrophys 37, 603-648 (1999).

[31] Joseph, C. L. and Woodgate, B. E., "UV Imaging Detectors: High-QE EBCMOS Enabling New Science Missions," in [American Astronomical Society Meeting Abstracts 217], Bulletin of the American Astronomical Society 43, 305.02 (Jan. 2011).

[32] Bianchi, L., Rodriguez-Merino, L., Viton, M., Laget, M., Efremova, B., Herald, J., Conti, A., Shiao, B., Gil de Paz, A., Salim, S., Thakar, A., Friedman, P. G., Rey, S.-C., Thilker, D., Barlow, T. A., Budavári, T., Donas, J., Forster, K., Heckman, T. M., Lee, Y.-W., Madore, B. F., Martin, D. C., Milliard, B., Morrissey, P., Neff, S. G., Rich, R. M., Schiminovich, D., Seibert, M., Small, T., Szalay, A. S., Wyder, T. K., Welsh, B. Y., and Yi, S. K., "Statistical Properties of the GALEX-SDSS Matched Source Catalogs, and Classification of the UV Sources," Astrophysical Journal, Supplement 173, 659-672 (Dec. 2007). 